\title{
Case report of a KIT-mutated melanoma patient with an excellent response to apatinib and temozolomide combination therapy
}

This article was published in the following Dove Press journal:

OncoTargets and Therapy

14 September 2017

Number of times this article has been viewed

\author{
Cong Luo' \\ Jiayu Shen ${ }^{2}$ \\ Jieer Ying ${ }^{\prime}$ \\ Xianhua Fang ${ }^{3}$ \\ Xiaohong Wang' \\ Zhixuan $\mathrm{Fu}^{4}$ \\ Peng Liu ${ }^{5}$ \\ 'Department of Abdominal Oncology, \\ Zhejiang Cancer Hospital, ${ }^{2}$ The \\ Second Clinical Medical College, \\ Zhejiang Chinese Medical University, \\ ${ }^{3}$ Department of Pathology, \\ ${ }^{4}$ Department of Colorectal Surgery, \\ ${ }^{5}$ Department of Radiotherapy, \\ Zhejiang Cancer Hospital, Hangzhou, \\ People's Republic of China
}

Correspondence: Cong Luo

Department of Abdominal Oncology,

Zhejiang Cancer Hospital, I East Banshan

Road, Gongshu, Hangzhou 310022 ,

People's Republic of China

Tel +86 I34 567| I894

Fax +86 57I 88I 22082

Email congluo939291@gmail.com
Abstract: Malignant melanoma is one kind of malignant disease which has high rates of mortality, metastasis, and poor prognosis. The therapeutic landscape is rapidly changing with the development of novel agents in recent decades, such as anti-PD-1 agents, anti-CTLA-4 agents, and BRAF inhibitors. However, since most of these novel agents are very expensive, not all patients can afford them. Apatinib is a novel oral small-molecule tyrosine kinase inhibitor targeting the intracellular domain of vascular endothelial growth factor receptor 2 (VEGFR-2) and may also be effective on Ret, c-KIT, and c-src. Temozolomide (TMZ) is a second-generation alkylating agent and a cytotoxic drug for melanoma treatment. In this work, we reported a case of metastatic melanoma with an excellent response to apatinib/TMZ combination therapy with progression-free survival for more than one year. This patient showed high expression of CD117, VEGFR-3, and KIT mutation in exon 11, suggesting that apatinib may induce clinical response via inhibiting VEGFR and c-KIT. Apatinib/TMZ combination therapy could be a new option for the treatment of advanced melanoma with KIT mutation.

Keywords: advanced melanoma, KIT mutation, apatinib, temozolomide, combination therapy

\section{Introduction}

Malignant melanoma (MM) has characteristics of high mortality, high metastasis, and drug resistance. MM accounts for only $4 \%$ of all dermatological malignancies, but it is responsible for about $80 \%$ mortality of skin tumors. Many melanoma patients exhibited genetic mutations including BRAF, NRAS, KIT, PTEN, GNAQ, GNA11, and CDKN2A mutations. ${ }^{1-3}$ Despite the fact that many novel agents (eg, anti-PD-1, anti-CTLA-4, and BRAF inhibitor) have promising effects on MM treatment while the response rate to cytotoxic agents is low in melanoma patients $(<20 \%)$ and many patients eventually become resistant to chemotherapy, chemotherapy is still the main palliative treatment for advanced MM in many countries due to its lower cost and accessibility. ${ }^{4-6}$ Temozolomide (TMZ) is a novel second-generation alkylating agent and an analog of dacarbazine (DTIC). The efficacy of TMZ is similar to that of DTIC, but TMZ provides improved quality of life and better physical function for the patients. ${ }^{4}$ It is recommended as the first-line therapy for MM in many countries due to its oral route of administration with nearly $100 \%$ bioavailability and extensive tissue distribution. ${ }^{4,5}$

In addition, MM is a highly angiogenic type of tumor, suggesting anti-angiogenesis as a way of treatment. ${ }^{7}$ Previous trials indicated that the combination of antiangiogenesis inhibitor and cytotoxic agent is a promising strategy for MM treatment, such as endostar with DTIC or bevacizumab with TMZ. ${ }^{8-11}$ 
Apatinib (YN968D1) is a novel potent oral smallmolecule tyrosine kinase inhibitor targeting the intracellular domain of vascular endothelial growth factor receptor 2 (VEGFR-2), and it may also be effective on Ret, c-KIT, and c-src. ${ }^{12}$ It has been proved that apatinib showed a survival benefit in gastric cancer and non-small-cell lung cancer. In the meantime, its benefit is under investigation in multiple tumor types (eg, breast cancer and liver cancer). Due to low side effects and improved outcomes, apatinib has a substantial potential to be a new therapeutic option in a variety of tumor types. Moreover, previous studies have shown that apatinib can reverse multidrug resistance (MDR), indicating a promising landscape of apatinib/cytotoxic agent combination therapy. ${ }^{13}$

In this study, we reported a promising case of a metastatic melanoma patient treated with apatinib plus TMZ as a third-line therapy.

\section{Case report}

On June 10, 2015, a 41-year-old man came to our hospital with a complaint of a 2-year history of enlarged right inguinal nodules after a melanoma surgery on his right planta pedis 4 years ago. The pathology diagnosis indicated MM with immunophenotype: HMB45 (++), S-100 (+), Vim (++), EMA (+), SMA (+), Ki-67 (+50\%), and Melan-A (+++). Except for multiple nodules in the right inguinal region, other metastases were ruled out by ultrasound, computed tomography $(\mathrm{CT})$ scan, and MRI. Since being evaluated as a stable disease (SD), after two cycles of chemotherapy (Table 1), he went through a lymph node dissection in a local hospital.

Unfortunately, no further treatment was taken after surgery due to patient's personal reasons. Three months after the surgery, chest CT scan showed multiple metastatic nodules in both lungs. Thus, a second-line chemotherapy of paclitaxel and carboplatin was then performed (Table 1). Because the patient complained of pelvic pain, early reexamination of CT and MRI was then performed 1 month after the first administration of the second-line chemotherapy.
The results showed multiple metastases in both lungs, hilar lymph nodes, liver, and bones (including vertebra, sternum, and iliac bone; Figure 1A and B), and it was considered to be a progressive disease (PD).

At this time, the patient progressed rapidly and was then refractory to traditional chemotherapy. Anti-PD-1 agents were unavailable, and the patient refused expensive targeted therapies and further clinical trials on MM in other hospitals. In the meantime, there is a medical products-donating project for apatinib that patients can get support since they pay for apatinib for the first 3 months. So after getting a written informed consent from the patient, a combination of apatinib and TMZ along with zoledronic acid was performed immediately (Table 1). After 2 months of treatment, the lesions in lung and liver were significantly reduced in size, which was considered to be a partial response (PR) based on the $\mathrm{CT}$ scan (Figure 1C and D). The following reviews of CT and MRI 3 months later showed no progression (Figure 1E and F). The patient continued to use apatinib and TMZ as a maintenance therapy. A progression-free survival (PFS) time of $>1$ year has been achieved.

The main toxicities the patient experienced were grade II hand-foot syndrome and grade II-III aphthous stomatitis. No other toxicities were complained and detected.

Next-generation sequencing (NGS; also known as highthroughput sequencing) analysis for the patient's blood sample after 11 months of this new treatment revealed KIT mutation in exon 11, but didn't show other genomic mutations, such as BRAF, NRAS, PTEN, or CDKN2A. The protein expressions of CD117 and VEGFR3 were high (Figure 2). As the patient experienced multiple drugs treatment that might change gene mutation, we further tested the inguinal lymph node tissue samples before the treatment. The result also revealed only KIT mutation in exon 11.

\section{Discussion}

Gene mutation plays a pivotal role in melanoma. Reported mutated genes include $B R A F, N R A S$, and KIT. KIT mutations

Table I Specific regimens and their efficacy

\begin{tabular}{|c|c|c|c|c|}
\hline Regimen & Beginning & Cycles & Evaluation & Efficacy \\
\hline $\begin{array}{l}\text { DTIC (400 mg/d DI-3) + cisplatin } \\
(45 \mathrm{mg} / \mathrm{d} \mathrm{DI}-3)+\text { endostar } \\
(15 \mathrm{mg} / \mathrm{d} \mathrm{DI}-\mathrm{l4}) \text { Q2ID }\end{array}$ & $\begin{array}{l}\text { After being diagnosed } \\
\text { of inguinal lymph } \\
\text { node metastasis }\end{array}$ & 2 & After 2 cycles of chemotherapy & SD \\
\hline $\begin{array}{l}\text { Paclitaxel ( } 270 \text { mg DI) + carboplatin } \\
(600 \mathrm{mg} \text { DI) Q2 ID }\end{array}$ & $\begin{array}{l}5 \text { months after first- } \\
\text { line chemotherapy }\end{array}$ & I & $\begin{array}{l}\text { I month after first administration of } \\
\text { second-line chemotherapy }\end{array}$ & PD \\
\hline $\begin{array}{l}\text { Apatinib (500 mg QD) + TMZ } \\
(200 \mathrm{mg} \text { DI-5, Q28D) }\end{array}$ & $\begin{array}{l}\text { After being evaluated } \\
\text { as progressive disease }\end{array}$ & - & $\begin{array}{l}\text { After } 2 \text { months' and } 5 \text { months' } \\
\text { administration of combination therapy }\end{array}$ & PR \\
\hline
\end{tabular}

Abbreviations: D, day(s); DTIC, dacarbazine; PD, progressive disease; PR, partial response; Q, every; SD, stable disease; TMZ, temozolomide. 

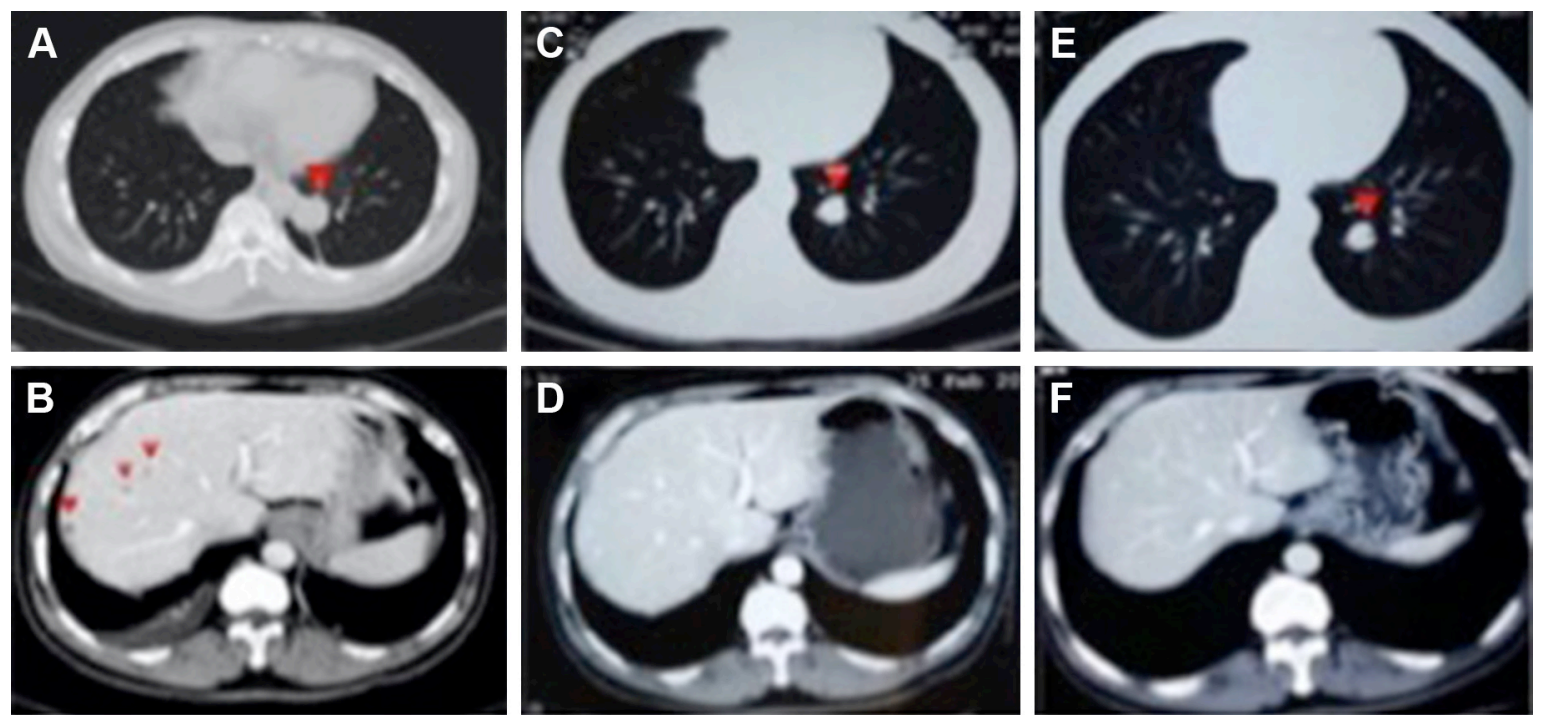

Figure I CT scan before and after apatinib/TMZ treatment (the lesions are indicated by red triangles): multiple metastases can be found in lungs and liver before apatinib/ TMZ therapy ( $\mathbf{A}$ and $\mathbf{B})$, and the lesions were smaller in lungs ( $\mathbf{C}$ and $\mathbf{E})$ after 2 months' administration of apatinib/TMZ and could barely be seen in liver ( $\mathbf{D}$ and $\mathbf{F})$ after 5 months of apatinib/TMZ treatment.

Abbreviations: CT, computed tomography; TMZ, temozolomide.

occur in about $3 \%$ of patients with melanoma, especially in acral, mucosal, and chronic sun damage melanoma. The most common KIT mutation identified in MM is located in exons $11(\sim 70 \%), 13$ (13\% or so), and 17 (9\% or so). As mentioned above, the patient with acral melanoma reported in this work had KIT mutation in exon 11, which may account for melanoma developing. KIT is found to be dispensable for growth, differentiation, migration, and proliferation in melanocytes systems. Besides, data show that abnormal activation of KIT signaling leads to increased growth and survival behavior and increased oncogenic potential in melanoma cells, indicating the reason why this patient progressed..$^{14,15}$

Regimens in melanoma treatment include checkpoint immunotherapy, BRAF-targeted therapy, high-dose interleukin-2, chemotherapy, biochemotherapy, and clinical trials. For those who have KIT mutation, imatinib is also a good option. Results from one trial of imatinib in Chinese patients harboring KIT mutation or amplification showed the median PFS of 3.5 months, and the median PFS for patients who had PR or SD versus PD was 9.0 versus 1.5 months. ${ }^{16}$ Apart from the regimens mentioned above, the landscape of anti-angiogenesis agents in MM treatment has aroused increasing attention. Results from a Phase II trial led by Chinese Professor Guo showed that median PFS in the endostar/DTIC arm was 4.5 months versus 1.5 months in placebo/DTIC arm $(p=0.013){ }^{8}$ There were statistically significant improvements in median overall survival (OS; 12.0 vs 8.0 months, $p=0.005$ ) in favor of endostar/DTIC arm.
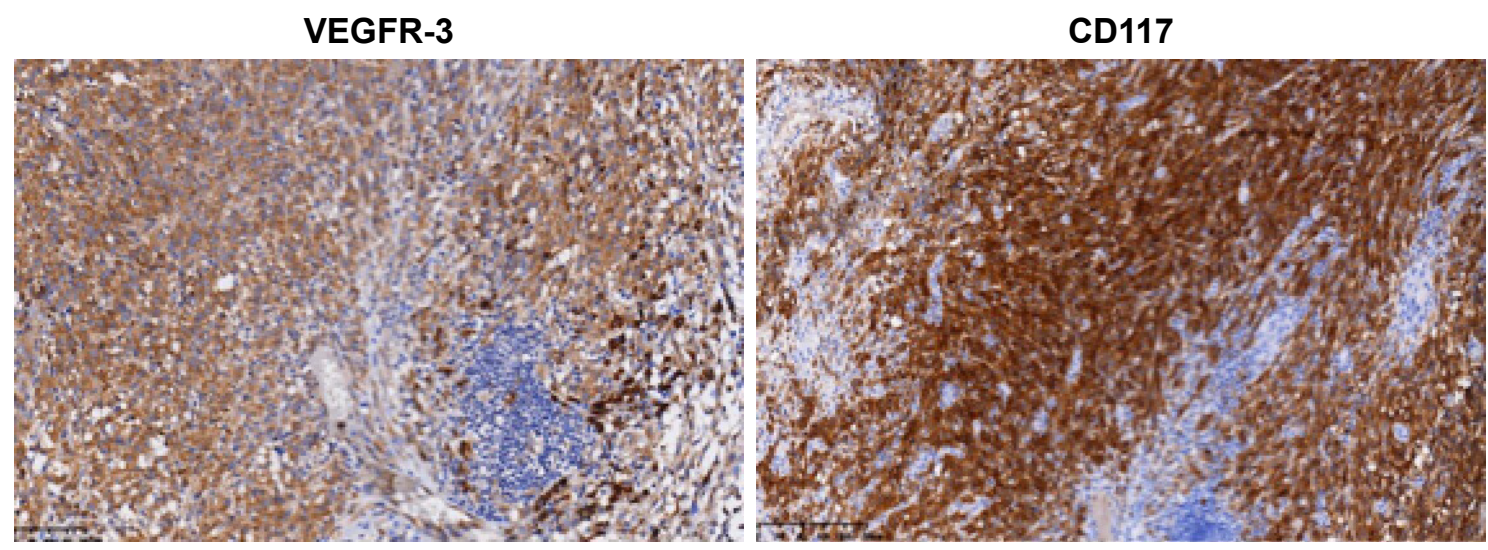

Figure 2 Immunohistochemical staining showing high expression of CDII7 and VEGFR-3. Magnification power: 100X. Abbreviation: VEGFR-3, vascular endothelial growth factor receptor 3. 
Based on this trial, the Chinese Society of Clinical Oncology recommends endostar plus DTIC as the first-line setting for advanced MM treatment. Besides, a number of Phase II clinical trials have shown the efficacy of bevacizumab/cytotoxic agent combination therapy in melanoma treatment. ${ }^{9-11}$ For example, SAKK50/07 trial evaluated the efficacy of bevacizumab/TMZ combination therapy in 62 patients, and the results showed that overall response rate was $16.1 \%$ (complete response: 1 patient and PR: 9 patients), and median PFS and OS were 4.2 and 9.6 months, respectively. ${ }^{11}$ All the information gave us a hint that the combination of antiangiogenesis inhibitor and cytotoxic agent could be effective on MM treatment.

Apatinib is an anti-angiogenesis agent that preferably targets VEGFR-2. Besides, one previous study has shown that apatinib could also potently inhibit the activities of Ret, c-KIT, and c-src. ${ }^{12}$ Multiple clinical trials have shown the survival benefit of apatinib in tumors such as gastric cancer and non-small-cell lung cancer. Apart from the ongoing trials, some case reports also showed the promising landscape of apatinib in various solid tumors such as advanced angiosarcoma and myxoid/round cell liposarcoma. ${ }^{17,18}$ It is also worth noticing that some preclinical studies show that apatinib can reverse MDR with other conventional antineoplastic drugs by inhibiting the efflux function of ABC transporters such as P-gp (ABCB1), MRP1 (ABCC1), and BCRP (ABCG2), suggesting that the combination of apatinib and chemotherapeutic agents may be a promising strategy in clinic. ${ }^{13}$

In this case, the patient with advanced melanoma failed two lines of chemotherapy, and he refused to accept expensive novel drugs. Therefore, after getting a written informed consent from the patient, we gave him a combination regiment of an anti-angiogenesis apatinib and one alkylating agent TMZ. Surprisingly, he showed a longer PFS of $>1$ year. Compared with the results of therapies mentioned above such as endostar plus DTIC, bevacizumab plus TMZ, and imatinib, our combination regiment showed better efficacy than TMZ or imatinib monotherapy or TMZ combination therapy reported previously. Besides, this treatment is also convenient for patients since both of them are oral drugs. In addition, the main adverse events were tolerable with mild hand-foot syndrome and aphthous stomatitis.

Considering that many melanoma patients have gene mutations and apatinib not only targets VEGFR but also works on Ret, c-KIT, and c-src, some tests were done to see whether there were any abnormalities in this patient. Because the sample of his right planta pedis was poorly stored and it took time to test every piece of the inguinal lymph node sample, we preferentially tested the patient's blood first hoping to find something. The patient's NGS analysis revealed only KIT mutation in exon 11 . It was really surprising to find KIT mutation since it was even rare in tumors with high prevalence of KIT mutation like gastrointestinal stromal tumor, let alone in melanoma with $<3 \%$ mutation rate of KIT. The reason why KIT mutation could be detected in blood was that tumor cells entered into the vessels and then released DNA, which explained how the patient developed metastasis. The excellent response in this patient with a KITmutant melanoma suggests that routine screening of acral melanoma for KIT mutation may be critical in identifying patients who may benefit from apatinib. Besides, tests of the inguinal lymph node sample were done later and revealed high expression of CD117 and VEGFR-3, suggesting that apatinib may work for the patient by inhibiting VEGFR and c-KIT. However, it still needs more large prospective trials to verify the efficacy and safety of this treatment.

\section{Conclusion}

In this case report, the combination of apatinib and TMZ exerted good efficacy and safety in the treatment of a melanoma patient with KIT mutation in exon 11, who had progressed after the second-line chemotherapy. As NGS analysis did not find any other gene mutations, KIT might be the patient's driver gene, suggesting that routine screening of acral melanoma for KIT mutation may open up new treatment choice for those patients. Considering apatinib has multiple mechanisms in anti-tumor effect, it is possible that apatinib plus TMZ might be a feasible option in metastatic melanoma with KIT mutation in exon 11. Large prospective trials are needed to confirm the efficacy and safety of apatinib and $\mathrm{TMZ}$ combination therapy for MM treatment in the future.

\section{Acknowledgments}

We thank the patient for his participation and his agreement and written informed consent for publication of his information and accompanying images in this case report. We also thank Burning Rock Biotech for doing NGS analysis. This research was supported by the National Natural Science Foundation of China (grant number 81372210) and the Natural Science Foundation of Zhejiang Province (grant number LY15H160003).

\section{Disclosure}

The authors report no conflicts of interest in this work. 


\section{References}

1. Zebary A, Omholt K, Vassilaki I, et al. KIT, NRAS, BRAF, and PTEN mutations in a sample of Swedish patients with acral lentiginous melanoma. J Dermatol Sci. 2013;72(3):284-289.

2. Van Raamsdonk CD, Griewank KG, Crosby MB, et al. Mutations in GNA11 in uveal melanoma. N Engl J Med. 2010;363(23):2191-2199.

3. Bishop DT, Demenais F, Goldstein AM, et al. Geographical variation in the penetrance of CDKN2A mutations for melanoma. J Natl Cancer Inst. 2002;94(12):894-903.

4. Middleton MR, Grob JJ, Aaronson N, et al. Randomized phase III study of temozolomide versus dacarbazine in the treatment of patients with advanced metastatic malignant melanoma. J Clin Oncol. 2000;18(1): $158-166$.

5. Kiebert GM, Jonas DL, Middleton MR. Health-related quality of life in patients with advanced metastatic melanoma: results of a randomized phase III study comparing temozolomide with dacarbazine. Cancer Invest. 2003;21(6):821-829.

6. Luo C, Shen J. Research progress in advanced melanoma. Cancer Lett. 2017;397:120-126.

7. Ugurel S, Rappl G, Tilgen W, Reinhold U. Increased serum concentration of angiogenic factors in malignant melanoma patients correlates with tumor progression and survival. J Clin Oncol. 2001;19(2):577-583.

8. Cui C, Mao L, Chi Z, et al. A phase II, randomized, double-blind, placebocontrolled multicenter trial of Endostar in patients with metastatic melanoma. Mol Ther. 2013;21(7):1456-1463.

9. Piperno-Neumann S, Diallo A, Etienne-Grimaldi MC, et al. Phase II trial of bevacizumab in combination with temozolomide as first-line treatment in patients with metastatic uveal melanoma. Oncologist. 2016;21(3): 281-282.
10. Kottschade LA, Suman VJ, Perez DG, et al. A randomized phase 2 study of temozolomide and bevacizumab or nab-paclitaxel, carboplatin, and bevacizumab in patients with unresectable stage IV melanoma: a North Central Cancer Treatment Group study, N0775. Cancer. 2013;119(3): 586-592.

11. von Moos R, Seifert B, Simcock M, et al. First-line temozolomide combined with bevacizumab in metastatic melanoma: a multicentre phase II trial (SAKK 50/07). Ann Oncol. 2012;23(2):531-536.

12. Tian S, Quan H, Xie C, et al. YN968D1 is a novel and selective inhibitor of vascular endothelial growth factor receptor-2 tyrosine kinase with potent activity in vitro, and in vivo. Cancer Sci. 2011;102(7): 1374-1380.

13. Mi YJ, Liang YJ, Huang HB, et al. Apatinib (YN968D1) reverses multidrug resistance by inhibiting the efflux function of multiple ATPbinding cassette transporters. Cancer Res. 2010;70(20):7981-7991.

14. Li G, Satyamoorthy K, Herlyn M. N-cadherin-mediated intercellular interactions promote survival and migration of melanoma cells. Cancer Res. 2001;61(9):3819-3825.

15. Smalley KS, Sondak VK, Weber JS. c-KIT signaling as the driving oncogenic event in sub-groups of melanomas. Histol Histopathol. 2009;24(5):643-650.

16. Guo J, Si L, Kong Y, et al. Phase II, open-label, single-arm trial of imatinib mesylate in patients with metastatic melanoma harboring c-KIT mutation or amplification. J Clin Oncol. 2011;29(21):2904-2909.

17. Ji G, Hong L, Yang P. Successful treatment of angiosarcoma of the scalp with apatinib: a case report. Onco Targets Ther. 2016;9:4989-4992.

18. Dong M, Bi J, Liu X, Wang B, Wang J. Significant partial response of metastatic intra-abdominal and pelvic round cell liposarcoma to a smallmolecule VEGFR-2 tyrosine kinase inhibitor apatinib: a case report. Medicine. 2016;95(31):e4368.
OncoTargets and Therapy

\section{Publish your work in this journal}

OncoTargets and Therapy is an international, peer-reviewed, open access journal focusing on the pathological basis of all cancers, potential targets for therapy and treatment protocols employed to improve the management of cancer patients. The journal also focuses on the impact of management programs and new therapeutic agents and protocols on

\section{Dovepress}

patient perspectives such as quality of life, adherence and satisfaction. The manuscript management system is completely online and includes a very quick and fair peer-review system, which is all easy to use. Visit http://www.dovepress.com/testimonials.php to read real quotes from published authors. 